\title{
Theoretical study of the thermal behavior of free and alumina-supported Fe-C nanoparticles
}

\author{
Aiqin Jiang ${ }^{1}$, Neha Awasthi ${ }^{1}$, Aleksey N. Kolmogorov ${ }^{1}$, Wahyu Setyawan ${ }^{1}$, \\ Anders Börjesson ${ }^{2}$, Kim Bolton ${ }^{2}$, Avetik R. Harutyunyan ${ }^{3}$ and Stefano Curtarolo ${ }^{1,4}$ \\ ${ }^{1}$ Department of Mechanical Engineering and Materials Science, \\ Duke University, Durham, NC 27r08, \\ ${ }^{2}$ University College of Boraas, SE-501 90, \\ Boraas, and Physics Department, \\ Göteborg University, SE-412 96, Göteburg, Sweden, \\ ${ }^{3}$ Honda Research Institute USA Inc., \\ 1381 Kinnear Road, Columbus, OH 43212, \\ ${ }^{4}$ corresponding author: stefano@duke.edu
}

(Dated: March 14, 2018)

\begin{abstract}
The thermal behavior of free and alumina-supported iron-carbon nanoparticles is investigated via molecular dynamics simulations, in which the effect of the substrate is treated with a simple Morse potential fitted to $a b$ initio data. We observe that the presence of the substrate raises the melting temperature of medium and large $\mathrm{Fe}_{1-x} \mathrm{C}_{x}$ nanoparticles $(x=0-0.16, N=80-1000$, nonmagic numbers) by 40-60 K; it also plays an important role in defining the ground state of smaller Fe nanoparticles $(N=50-80)$. The main focus of our study is the investigation of Fe-C phase diagrams as a function of the nanoparticle size. We find that as the cluster size decreases in the 1.1-1.6-nm-diameter range the eutectic point shifts significantly not only toward lower temperatures, as expected from the Gibbs-Thomson law, but also toward lower concentrations of C. The strong dependence of the maximum $\mathrm{C}$ solubility on the $\mathrm{Fe}-\mathrm{C}$ cluster size may have important implications for the catalytic growth of carbon nanotubes by chemical vapor deposition.
\end{abstract}

\section{INTRODUCTION}

Catalytic chemical vapor deposition (CVD) is a widely used method for the production of carbon nanotubes (CNTs) by decomposition of hydrocarbons (such as $\mathrm{CH}_{4}$, $\mathrm{C}_{2} \mathrm{H}_{2}$ etc.) or carbon monoxide on supported metal catalysts (Fe, Ni, Co, FeMo, etc.) [1, 2, 3, 4, 5]. Despite numerous studies, the growth mechanism of nanotubes is still not well understood. Among the most studied factors that control the growth process are the kinetics of carbon transport [2, 3, 4, 5, 6, 6, 8, 8, 9, 10, the thermodynamics of the catalyst particles 11, 12, 13, 14, 15], and their interaction with substrates (oxides or zeolites) 16, 17, 18.

The vapor-liquid-solid (VLS) model [19] for the CNT growth by CVD implies that the catalytic particle should be in a liquid state which allows rapid diffusion of carbon atoms throughout the particle. The bulk diffusion of carbon through the metal nanoparticle is driven by concentration gradients [20], and it is considered to be the rate-limiting step in the growth of filaments or carbonaceous deposits [21, 22]. The activation energies $(\sim 1.2-1.8$ $\mathrm{eV}$ ) measured for thermal CVD growth of nanofibers or nanotubes are consistent with those for the carbon diffusion through the corresponding metals [2, 3, 4, 22, 23], hence further supporting the bulk diffusion VLS model. Another mechanism, the surface-mediated carbon transport model, has been proposed [6, 7]. The low temperature nanotube synthesis by plasma-enhanced CVD 8, 9, 10, 16, 24, 25] implies that the catalyst could be in a solid state. However, the temperature of the ac- tive nanoparticles is extremely difficult to measure during the growth process. In fact, the bombardment of energetic species in plasma and the exothermic dehydrogenation reactions of hydrocarbon can increase the local temperature of the catalyst, even if the substrate is kept in thermal equilibrium. This phenomenon can promote the surface melting of the nanoparticle and, concurrently, facilitate the growth of the nanotube [13, 14].

Because the overall catalytic capability of nanoparticles strongly depends on whether they are in the liquid or solid states, their thermal behavior has been extensively investigated with experimental $11,12,26,27,28$, 29, 30, 31, 32 and theoretical means [13, 14, 33, 34, 35, 36, 37, 38, 39, 40]. Under CVD experimental conditions, the melting temperatures of catalytic particles are strongly reduced because of the dissolved carbon (liquidus and solidus slopes in the metal-C phase diagram) and the relatively high surface energy with respect to the bulk materials (Gibbs-Thomson phenomenon). Unusually low melting points of $600-700^{\circ} \mathrm{C}$ have been observed for oversaturated solutions of carbon (up to 50 at. $\%$ at $700^{\circ} \mathrm{C}$ ) in $\mathrm{Fe}, \mathrm{Ni}$, and $\mathrm{Co}$ metals 31. In these cases, the fluidization of the metal catalytic particles at low or moderate temperature was attributed to the creation of highly dispersed unstable solutions oversaturated with carbon, with concentrations well above the limit of the stable carbide [32, 41].

With so many factors influencing the catalytic growth of $\mathrm{CNT}$, the role of the substrate on the thermal properties of the particle is often overlooked. In fact, there are experimental indications that the presence of substrate could be an important factor in the thermodynamics of 
the particle and in regulating the growth of nanotubes [16, 17, 18]. Most of the theoretical research has been focused on the melting behavior of free-standing pure [14, 33, 34, 35] or bimetallic [36, 37, 38, 39, 40] clusters. A phase diagram of free-standing Fe-C clusters of fixed size $(\sim 2.4 \mathrm{~nm})$ has been recently calculated 13,42$]$. There have been investigations performed on supported catalysts [15, 38, 43, 44], showing that the cluster-substrate interaction strongly affects the melting temperature of particles and thus could influence the nanotube growth rate. A more general picture, capturing the particlesubstrate interaction and size effects on the phase diagram, is still lacking and is the main subject of our study.

In this paper we investigate thermal behaviors for free and alumina-supported Fe-C nanoparticles with molecular dynamics (MD) simulations. In Section II we describe the $a b$ initio development of a simple interaction between $\mathrm{Fe}$ and $\mathrm{Al}_{2} \mathrm{O}_{3}$ which is essential for the calculations of supported clusters of reasonable sizes. In Section III, by using MD simulations we explore the thermodynamics of $\mathrm{Fe}$ nanoclusters and the phase diagrams of Fe$\mathrm{C}$ binary nanoparticles focusing on the effects of cluster's size and interfacial interactions on the thermal properties (Fe-C up to $\sim 16 \%$ carbon concentration). In the same Section we show that not only the eutectic temperature but also the eutectic composition are size- and substratedependent. Section IV is devoted to the exploration of the peculiar characteristics of very small clusters. Conclusions are described in Section V.

\section{THE FE- $\mathrm{AL}_{2} \mathrm{O}_{3}$ POTENTIAL}

In this section we model the many-body $\mathrm{Fe}-\mathrm{Al}_{2} \mathrm{O}_{3}$ interaction with a simple classical potential. If the calculation of this interaction energy involved the summation over all substrate atoms (e.g. using modified charge transfer potential [45, 46]) the simulation would be computationally very expensive (a 200-atom nanoparticle would require a 1000-atom substrate patch). Ideally, a suitable potential for MD simulations would depend on three integral variables, or even one (the distance from an iron atom to the surface) if the potential corrugation is small. The validity of such simplification critically depends on how weak the Fe- $\mathrm{Al}_{2} \mathrm{O}_{3}$ interaction is compared to those of $\mathrm{Fe}-\mathrm{Fe}$ and $\mathrm{Al}_{2} \mathrm{O}_{3}-\mathrm{Al}_{2} \mathrm{O}_{3}$.

There are three possible terminations for (0001) $\alpha$ $\mathrm{Al}_{2} \mathrm{O}_{3}$ surfaces [47, 48, 49, 50]: stoichiometric Alterminated, Al-Al-terminated (two top Al layers), and O-terminated. These are depicted in Figure 1(a). Theoretical calculations have predicted that the most stable surface is the stoichiometric one, terminated by a single layer of $\mathrm{Al}$ [47]. This type of surface is also believed to be the most often observed under ultra high vacuum conditions 48, 49, 50]. Therefore in this work we will restrict our analysis of Fe over $\mathrm{Al}_{2} \mathrm{O}_{3}$ using only the stable stoichiometric Al-termination.

\section{A. Details of Calculation}

To develop the interaction between Fe clusters and $\mathrm{Al}_{2} \mathrm{O}_{3}$ surfaces, we perform density functional theory $a b$ initio calculations with Vienna Ab-Initio Simulation Package VASP [51, 52] with projector augmented waves (PAW) [53, 54] and exchange-correlation functionals as parameterized by Perdew, Burke, and Ernzerhof (PBE) [55] for the generalized gradient approximation (GGA). Simulations are carried out at zero temperature, with spin polarization and without zero-point motion. We use an energy cutoff of $500 \mathrm{eV}$ and a $6 \times 6 \times 1 \mathrm{k}$-point Monkhorst-Pack mesh 56]. The force tolerance for structural relaxation is set to $0.1 \mathrm{eV} / \AA$. The unit cell is hexagonal with lattice parameters $a=4.767$ and $c=29.143$ $\AA$. The length of the lattice vector normal to surface is kept large enough to minimize the neighboring supercell interaction. Vertically, there is at least $12 \AA$ of empty space. In addition, a dipole layer is applied in $z$-direction to minimize electrostatic effects. A schematic of the unit cell is shown in Figure 1(b).

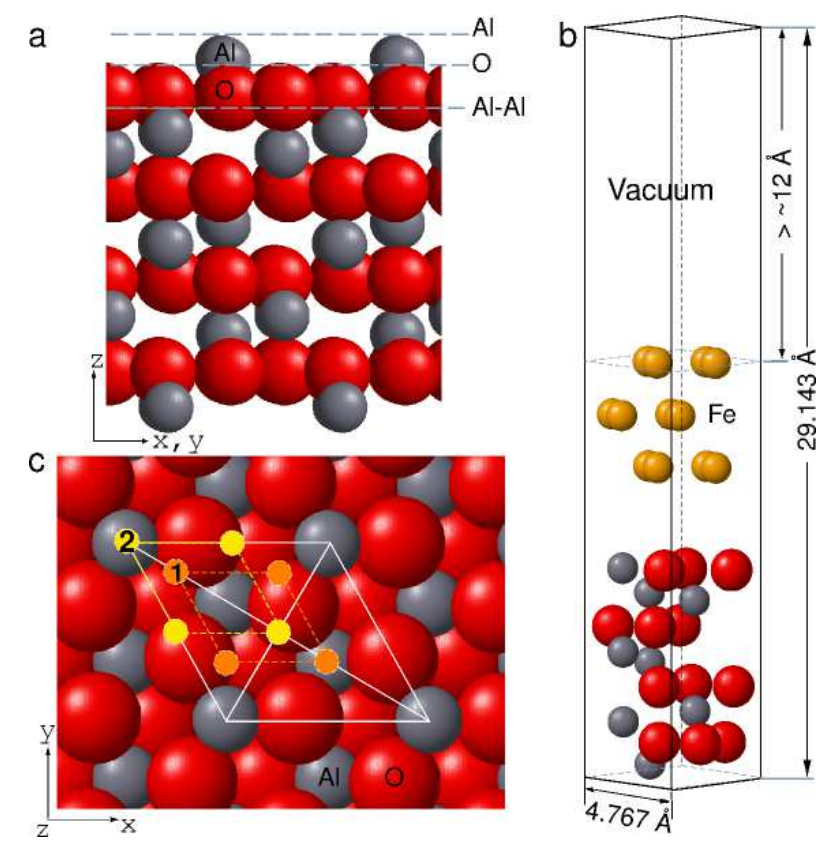

FIG. 1: (color online). (a) Possible surface terminations of $\alpha$ $\mathrm{Al}_{2} \mathrm{O}_{3}$ (side view). (b) Schematic of the hexagonal unit cell. (c) The two different high symmetry positions "1" and "2" of the inner layer of adsorbed iron (top view), each Fe layer has four atoms.

\section{B. Results and Discussion}

The calculated lattice parameters of the bulk alumina (space-group \# 167) with a hexagonal unit cell (hR30), $a=4.767 \AA$ and $c=12.994 \AA$, are in good agreement with the experimental values, $a_{\exp }=4.742 \AA$ and $c_{\text {exp }}=12.919 \AA$ [57, 58]. Our $\alpha-\mathrm{Al}_{2} \mathrm{O}_{3}$ slab consists of 
four oxygen layers as shown in Figure 1(b). The structure is relaxed from the bulk $\alpha-\mathrm{Al}_{2} \mathrm{O}_{3}$ configuration while keeping one oxygen and one aluminum layers at the bottom frozen. The surface undergoes considerable relaxations: the interlayer spacings for the top four layers become $d=0.117,0.899,1.019$ and $0.264 \AA$, differing from the corresponding bulk values by $-87.3 \%, 7.8 \%,-47.1 \%$, and $22.1 \%$, respectively. These values compare well with the previous 18 oxygen-layer simulations $(-87.4 \%, 3.1 \%$, $41.7 \%, 18.9 \%$ ) [47], suggesting that an alumina slab with four layers of oxygen is thick enough to be a realistic model of the $\mathrm{Al}_{2} \mathrm{O}_{3}$ surface. On the top of the relaxed $\alpha$ $\mathrm{Al}_{2} \mathrm{O}_{3}$ substrate we place a few close-packed layers of iron while keeping the in-plane lattice vectors of Fe identical to the bulk value of alumina. Because of the fortunate match between the natural Fe and the $\alpha-\mathrm{Al}_{2} \mathrm{O}_{3}$ surface lattice spacings (within 3\%), the effect of the interface strain energy on the total binding is insignificant. Closepacked iron layers can be put in different ways on top of alumina. Thus, we consider the two high symmetry positions labeled as "1" and "2" in Figure 1(c).

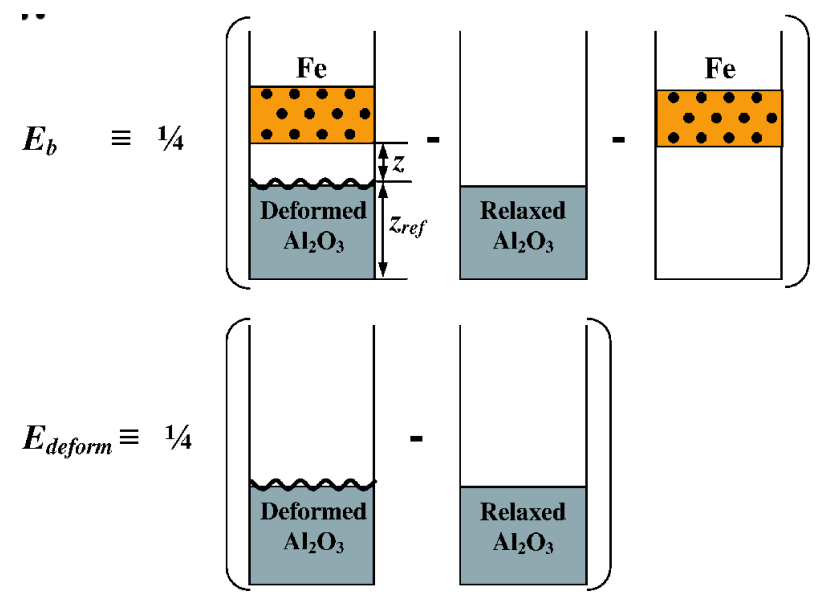

FIG. 2: (color online). The definition of the binding and deformation energies.

The binding energy between one Fe column (one Fe per layer) and $\mathrm{Al}_{2} \mathrm{O}_{3}$ is defined as

$$
E_{b} \equiv \frac{1}{4}\left[E_{\mathrm{Fe}-\mathrm{Al}_{2} \mathrm{O}_{3}}-E_{\text {relaxed }\left[\mathrm{Al}_{2} \mathrm{O}_{3}\right]}-E_{\mathrm{Fe}}\right],
$$

where $E_{\mathrm{Fe}-\mathrm{Al}_{2} \mathrm{O}_{3}}$ is the total energy of the optimized system, $E_{\text {relaxed }\left[\mathrm{Al}_{2} \mathrm{O}_{3}\right]}$ and $E_{\mathrm{Fe}}$ are the energies of the relaxed $\mathrm{Al}_{2} \mathrm{O}_{3}$ slab and Fe layers, respectively (note that the factor $1 / 4$ appears because there are four $\mathrm{Fe}$ per adsorbed layer). The presence of adsorbed Fe strongly modifies the alumina surface, and the deformation of the surface may affect the total binding considerably. To evaluate this effect, we define the substrate deformation energy as

$$
E_{\text {deform }} \equiv \frac{1}{4}\left[E_{\text {deformed }\left[\mathrm{Al}_{2} \mathrm{O}_{3}\right]}-E_{\text {relaxed }\left[\mathrm{Al}_{2} \mathrm{O}_{3}\right]}\right],
$$

where $E_{\text {deformed }\left[\mathrm{Al}_{2} \mathrm{O}_{3}\right]}$ is the energy of an artificially isolated alumina slab with the same geometry as that of the optimized $\mathrm{Fe}-\mathrm{Al}_{2} \mathrm{O}_{3}$ system but without adsorbed iron. Figure 2 shows a schematic of the definitions of the two energies, $E_{b}$ and $E_{\text {deform. }}$. In our calculations only the surface is allowed to relax: iron atoms are kept at their ideal positions because we assume that a reliable $\mathrm{Fe}-\mathrm{Fe}$ interaction (e.g. Born-Mayer potential [59, 60]) will be able to properly describe their energetics.

To determine how many layers of Fe must be included in the calculations, we evaluate the $\mathrm{Fe}-\mathrm{Al}_{2} \mathrm{O}_{3}$ interaction for systems with different number of Fe layers situated at the same distance above the alumina surface. As summarized in Table【 the binding energy converges reasonably well once the iron film contains three or more layers. Therefore, we limit our calculations to the absorption of only three Fe layers. In addition, due to the corrugation of the surface, the binding energy depends on where the adsorbants are positioned in the $x$ - $y$ plane. Figure 1(c) shows the two high symmetry configurations of the inner layer of adsorbed film. The corrugation, calculated as the difference between the binding energies of the most and least stable configurations (position "1" and "2" in Figure 1(c), respectively), is less than $15 \%$ of the total energy. Such little sensitivity to the lateral position of iron allows us to consider the surface essentially flat.

\begin{tabular}{c|c}
\hline \hline $\begin{array}{c}\text { Number of Fe layers } \\
\text { (4 Fe per layer) }\end{array}$ & $E_{b}(\mathrm{meV})$ \\
\hline One & -666 \\
Two & -258 \\
Three & -160 \\
Four & -159 \\
Five & -146 \\
\hline \hline
\end{tabular}

TABLE I: The dependence of the binding energy on the thickness of close-packed Fe structure with the closest layer located in position "1" of Figure 1(c).

Figure 3(a) shows the binding $\left(E_{b}\right)$ and deformation $\left(E_{\text {deform }}\right)$ energies as a function of the distance $z$ from the surface. Since the $\mathrm{Al}_{2} \mathrm{O}_{3}$ slab experiences surface rearrangement, $z$ must be defined with respect to a fixed reference, in our case, the bottom of the unit cell (see Figure 21). For convenience, we use a constant shift $z_{\text {ref }}$ to have the minimum of the interaction energy at a reasonable distance of $2.25 \AA$. The strong contribution of $E_{\text {deform }}$ to $E_{b}$, shown in Figure 3(a), is caused by the considerable rearrangement of the surface atoms to accommodate the absorbed iron. The origin of the nonmonotonic variation of the deformation energy is clarified in Fig. 3(b), in which we show the vertical shifts of the outer $\mathrm{Al}$ and $\mathrm{O}$ layers, $z^{A l}$ and $z^{O}$, with respect to the $\mathrm{O}$ position when $\mathrm{Fe}$ is not present $(z \rightarrow \infty)$. The outer positively charged $\mathrm{Al}$ layer first rises above the substrate to be closer to the approaching Fe film and accept more charge (as has been shown previously for the $\mathrm{Zr}$ - 
terminated $\mathrm{Ni} / \mathrm{ZrO}_{2}$ system [61], the charge transfer between the outer $\mathrm{Zr}$ and $\mathrm{Ni}$, as well as the accumulation of charge in the gap between them, is significant). The maximum rise for the $\mathrm{Al}$ layer happens at the interlayer Al-Fe distance of $2.7 \AA(z=2.8 \AA$ in Fig. 3(b)), which corresponds to the kink in the deformation energy curve. After that point, both $\mathrm{Al}$ and $\mathrm{O}$ layers are pushed downwards. For $z<1.5 \AA$, one can also expect noticeable deformation of the $\mathrm{Fe}$ film, and the decomposition of the total $\mathrm{Fe}$ binding into the independent $\mathrm{Fe}-\mathrm{Fe}$ and $\mathrm{Fe}-\mathrm{Al}_{2} \mathrm{O}_{3}$ contributions may not be accurate anymore.

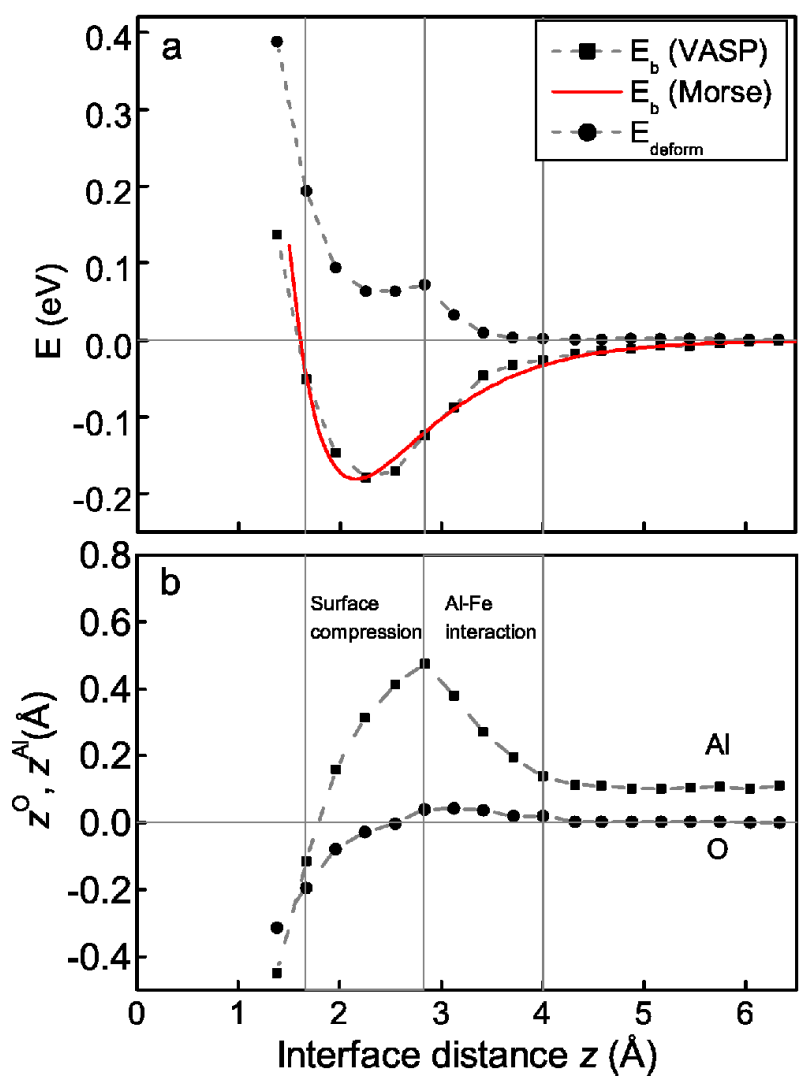

FIG. 3: (color online). (a) The binding energy of Fe- $\mathrm{Al}_{2} \mathrm{O}_{3}$ ( $a b$ initio values with fitted Morse potential) and deformation energy of the $\mathrm{Al}_{2} \mathrm{O}_{3}$ slab as functions of the distance from the surface. (b) Vertical coordinates of the outer $\mathrm{Al}$ and $\mathrm{O}$ layers of $\mathrm{Al}_{2} \mathrm{O}_{3}$ as function of the distance from the surface.

The $a b$ initio binding energy can be decomposed as a sum of contributions from each atom in the Fe "column" (assuming that the many-body Fe-Fe effects are appropriately described by an external interaction):

$$
E_{b}=\sum_{i=1}^{3} E_{i}
$$

where the energy per atom $E_{i}$ is taken to be a simple Morse potential [62] as:

$$
E_{i}=D\left\{\exp \left[-2 \alpha\left(z_{i}-z_{0}\right)\right]-2 \exp \left[-\alpha\left(z_{i}-z_{0}\right)\right]\right\}
$$

Fitting gives $D=153 \mathrm{meV}, \alpha=1.268 \AA^{-1}$, and $z_{0}=$ $2.219 \AA$. Figure 3 (a) shows the fit of $E_{b}$ calculated as a sum of three Morse interactions.

The strength of the Fe- $\mathrm{Al}_{2} \mathrm{O}_{3}$ interaction $(153 \mathrm{meV})$ amounts to only $\sim 10 \%$ of the Fe-Fe interlayer binding energy ( $\sim 1.24-1.42 \mathrm{eV}$, depending on the thickness of the Fe film), which implies that the latter is not significantly affected by the presence of the substrate. Hence, the assumption of decomposing the total Fe binding into the separate $\mathrm{Fe}-\mathrm{Fe}$ and $\mathrm{Fe}-\mathrm{Al}_{2} \mathrm{O}_{3}$ interactions is justified $a$ posteriori. Our simple $\mathrm{Fe}-\mathrm{Al}_{2} \mathrm{O}_{3}$ Morse interaction naturally incorporates the whole alumina surface deformation, since the binding energy has been defined with respect to an ideal $\mathrm{Al}_{2} \mathrm{O}_{3}$ slab and an isolated Fe film, as described in equation (11) and Figure 2, Within this framework, MD simulations on supported particles can be efficiently performed with reasonable accuracy by considering the $z$ component of the particle interaction with the flat substrate.

As we are interested in the properties of binary $\mathrm{Fe}_{1-x} \mathrm{C}_{x}$ nanoparticles, we need to evaluate the interaction between carbon surrounded by iron and the alumina substrate (Fe-embedded-C- $\mathrm{Al}_{2} \mathrm{O}_{3}$ interaction). Starting from three layers of adsorbed $\mathrm{Fe}$, we substitute one $\mathrm{Fe}$ with one $\mathrm{C}$ atom bringing the concentration to $x \sim 8.3 \%$. Depending on the position in which the substitution takes place, the binding energy $E_{b}\left[\mathrm{Fe}_{11} \mathrm{C}_{1}-\mathrm{Al}_{2} \mathrm{O}_{3}\right]$ fluctuates while remaining similar to $E_{b}\left[\mathrm{Fe}_{12}-\mathrm{Al}_{2} \mathrm{O}_{3}\right]$, which suggests some importance of many-body effects in the Feembedded-C- $\mathrm{Al}_{2} \mathrm{O}_{3}$ interaction. Because the substrate represents only a small perturbation to the total binding of the nanoparticle and the concentration of $\mathrm{C}$ in our simulations is small, we choose to treat the $\mathrm{C}-\mathrm{Al}_{2} \mathrm{O}_{3}$ and $\mathrm{Fe}-\mathrm{Al}_{2} \mathrm{O}_{3}$ interactions in the same way, i.e. we use the Fe$\mathrm{Al}_{2} \mathrm{O}_{3}$ Morse potential for both atom types. The validity of this approximation has been addressed by performing MD simulations, similar to those described in Section III, but here we vary the Morse parameters for the C- $\mathrm{Al}_{2} \mathrm{O}_{3}$ interaction. Our tests for supported $\mathrm{Fe}_{300} \mathrm{C}_{30}$ nanoparticles reveal that the thermodynamics of the systems is not very sensitive to the particular value of the $\mathrm{C}-\mathrm{Al}_{2} \mathrm{O}_{3}$ binding. For example, increasing or decreasing the strength of the $\mathrm{C}-\mathrm{Al}_{2} \mathrm{O}_{3}$ interaction for the $\mathrm{Fe}_{300} \mathrm{C}_{30}$ nanoparticles by a factor of two changes the melting temperature by $1-2 \%$, the amount comparable to the statistical error of the melting temperature determination in our simulations(see Section III). We conclude that the simple approximation of employing the same description for the $\mathrm{Fe}$ and $\mathrm{C}$ interactions with the substrate is suitable for this study.

\section{MOLECULAR DYNAMICS}

Among the taxonomy of thermodynamics phenomena for nanoparticles, melting has been subject of considerable interest. The characteristics of the melting process depend on a variety of parameters such as size [26, 63] 
and shape of the particles [64], concentration of impurities [65], and presence of substrates [43, 44].

In this section we are interested in the effect of size, carbon presence and $\mathrm{Al}_{2} \mathrm{O}_{3}$ substrate on the melting and solidification of binary Fe-C nanoparticles. We address these tasks by analyzing, with classical MD simulations, the liquidus and solidus lines of their phase diagrams, in function of the aforementioned parameters.

\section{A. Methods}

Molecular Dynamics Simulations. MD simulations are carried in the $N V T$ ensemble using the Verlet algorithm [66, 67] with a time step $\Delta t=1.0 \mathrm{fs}$. Of the several methods developed for controlling the temperature in MD simulations 68, 69, 70, 71, 72], Berendsen and Nosé-Hoover thermostats are most commonly used. In Fig. 4 we compare the temperature dispersion $\Delta T$ of the two thermostats with respect to that of the canonical distribution $\Delta T_{\text {canonical }}[73$ ] at $T=400$ $\mathrm{K}$ for small nanoparticles $\left(N_{F e}=50\right)$. We observe that the widely used Berendsen thermostat is more sensitive to the choice of the coupling constant. Inset in Figure 4 shows the distribution of instantaneous kinetic temperature for Berendsen thermostat for the typical value of $\Delta t / \tau=0.1$ [74], the Nosé-Hoover thermostat for $\tau$ $=25 \mathrm{fs}$, and the canonical distribution at $T=400 \mathrm{~K}$. The Nosé-Hoover thermostat $\left(\sigma_{T} \sim 47 \mathrm{~K}\right)$ reproduces the canonical distribution $\left(\sigma_{T} \sim 46 \mathrm{~K}\right)$ much better than the Berendsen thermostat $\left(\sigma_{T} \sim 17 \mathrm{~K}\right)$, making it a better choice for our constant temperature simulations.

Interatomic interaction. $\mathrm{Fe}-\mathrm{Fe}, \mathrm{Fe}-\mathrm{C}$, and $\mathrm{C}-\mathrm{C}$ interactions are described by Born-Mayer [59, 60], Johnson [75, 76] and Lennard-Jones [77] potentials, respectively. These interaction models are discussed in detail elsewhere [76]. The Morse potential, introduced in Section III, is used to model the Fe- $\mathrm{Al}_{2} \mathrm{O}_{3}$ interaction; the Fe-embedded-C- $\mathrm{Al}_{2} \mathrm{O}_{3}$ interaction ( $\mathrm{C}$ is diffused in $\mathrm{Fe}$ ) is taken to be identical to $\mathrm{Fe}-\mathrm{Al}_{2} \mathrm{O}_{3}$ as discussed before.

Initial configurations. To avoid excessive temperature fluctuations in the MD simulations of the nanoparticles one should start from the most stable configurations. We search for the best possible energy minima by randomly arranging atoms in a spherical nanoparticle, carefully optimizing the positions of iron and carbon atoms and finally annealing the nanoparticles for $6 \times 10^{6}$ MD iterations $(6 \mathrm{~ns})$. The annealing is performed in the following way: the nanoparticle is first heated to high temperature (from $1000 \mathrm{~K}$ to $1400 \mathrm{~K}$ depending on the size of the particle) for $0.6 \times 10^{6}$ steps, kept at constant temperature for another $0.6 \times 10^{6}$ iterations, and finally cooled to $0 \mathrm{~K}$ during the remaining $4.8 \times 10^{6} \mathrm{MD}$ steps.

Definition and determination of melting temperature. In our work, the melting phenomenon is analyzed by performing several MD simulations starting at about $300 \mathrm{~K}$ below the expected melting point with temperature increments of $10 \mathrm{~K}$ for small $(N<100)$ and $20 \mathrm{~K}$ for large clus-

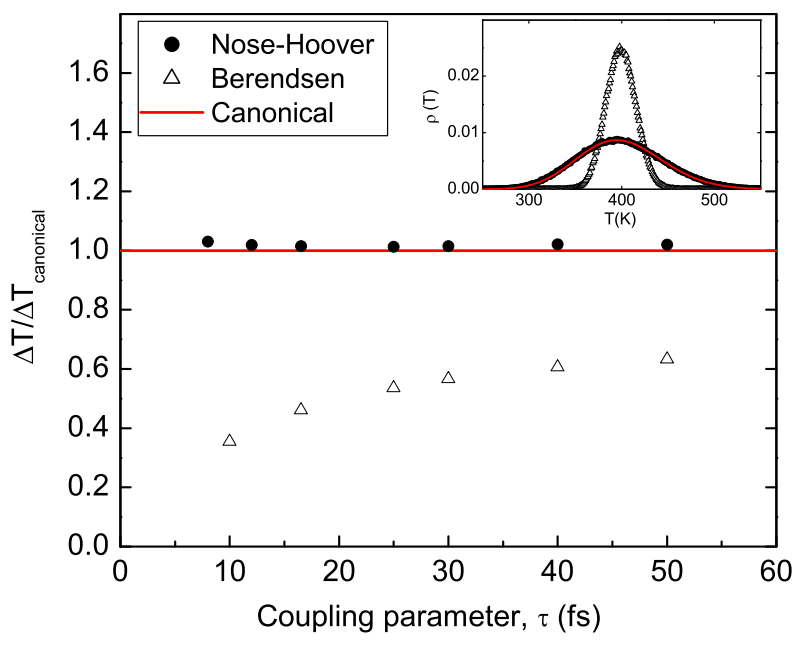

FIG. 4: (color online). Comparison of Berendsen and NoséHoover thermostat for $N_{F e}=50$ at $400 \mathrm{~K}$ for $\Delta t=1 \mathrm{fs}$ : temperature dispersion for Nosé-Hoover is closer to the canonical dispersion. Inset shows the distribution of instantaneous kinetic temperature for Berendsen thermostat for $\Delta t / \tau=0.1$, Nosé-Hoover thermostat for $\tau=25 \mathrm{fs}$, and the canonical distribution.

ters (with $5 \mathrm{~K}$ upon approaching the transition). Only the lowest temperature simulations begin from the annealed initial structures: the others start from the final configurations (positions, forces, velocities) of the preceding temperature simulation. Data gathering of the energies and other averages are performed over $10^{6} \mathrm{MD}$ steps.

Several dynamical and structural properties such as total energy, Lindemann index, diffusion coefficients, and pair correlation functions can be used to identify phase transitions in nanoparticles 78, 79]. Here, melting is characterized by the temperature dependence of the total energy (caloric curve), by the change in the total energy with time, and by the variation of the Lindemann index with respect to temperature [80]. The Lindemann index $\delta$ represents the root-mean-square relative bond-length fluctuation:

$$
\delta \equiv \frac{2}{N(N-1)} \sum_{i<j} \frac{\sqrt{\left\langle r_{i j}^{2}\right\rangle-\left\langle r_{i j}\right\rangle^{2}}}{\left\langle r_{i j}\right\rangle},
$$

where $r_{i j}$ is the distance between atom $i$ and $j, N$ is the number of particles and the average is calculated over an $\mathrm{MD}$ run at a given $T$. The melting point, which defines the temperature at which a solid becomes liquid, is a macroscopic concept for pure and bulk systems. Both finite-size and presence of more than one atomic species make the melting transition a continuous phenomenon that occurs over a range of temperatures, $\Delta T_{m}$, in which solid and liquid phases coexist with dif- 

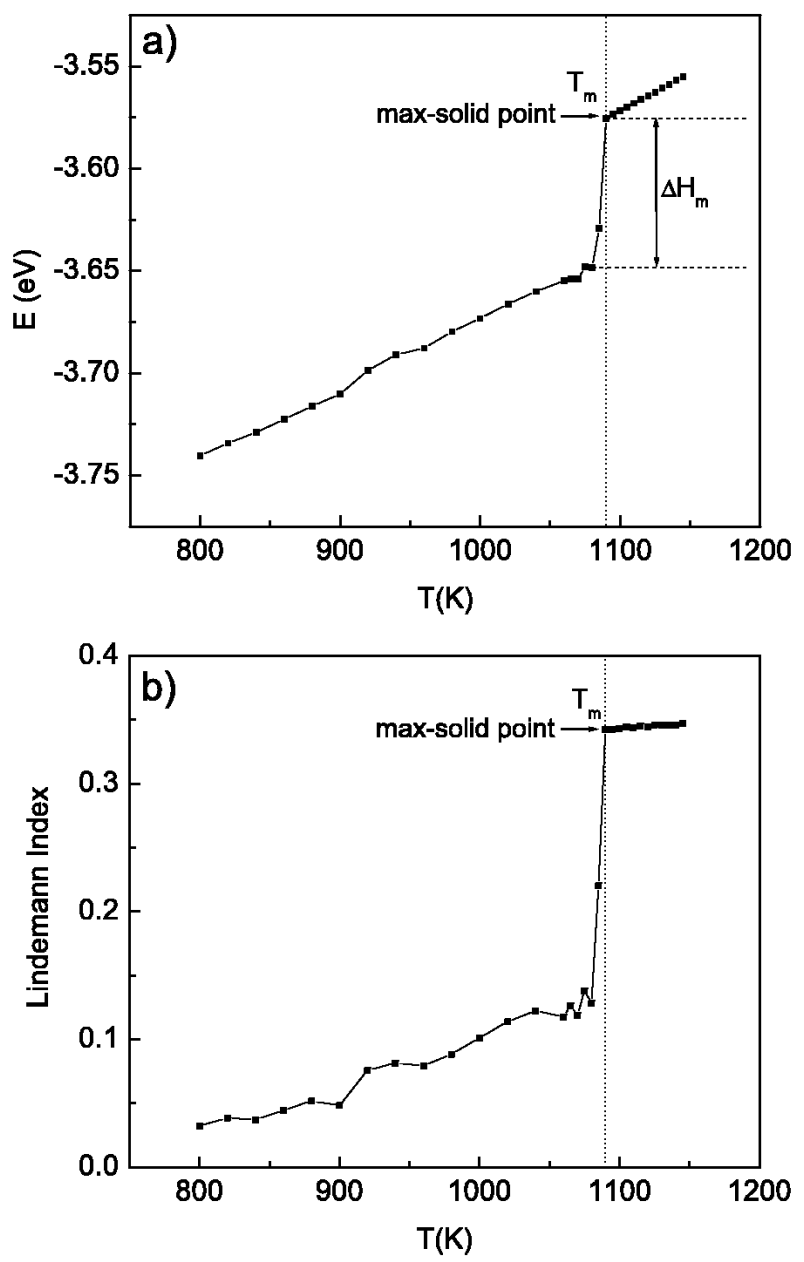

FIG. 5: Estimation of the melting temperature $T_{m}$ as $\max$ solid point for $\mathrm{Fe}_{400}$ nanoparticle from the caloric curve (panel a) and from the Lindemann index plot (panel b).

ferent fractions $81,82,83]$. To have a specific value of $T_{m}$ instead of a range, we define the melting temperature $T_{m}$ as the "max-solid point" which represents the maximum temperature at which the solid and the liquid phases coexist (the locus of all the max-solid points is the liquidus). Above $T_{m}$, no solid phase is present. Note that within this definition of $T_{m}$, we also identify plasticviscous nanoparticles as "liquid" 84]. Figure 5 shows an example of $T_{m}$ calculated from the caloric curve (panel a) and from the Lindemann index (panel b). Similarly, the min-liquid point is the minimum temperature at which the solid and the liquid phases coexist (the locus of all the min-liquid points is the solidus). The difference between the energies of the particle at the max-solid and at the min-liquid points defines the enthalpy of melting $\Delta H_{m}$.

\section{B. Melting of nanoparticles}

With the aforementioned method, we investigate pure nanoparticles of size $N_{F e}=80-1000$ atoms (diameter $d \sim 1-3 \mathrm{~nm}$ ). Caloric curves for particles with $N_{F e}>100$ show small melting intervals $\left(\Delta T_{m} \lesssim 10 \mathrm{~K}\right)$. For smaller clusters $\left(\mathrm{N}_{F e} \sim 80-100\right.$ atoms, $\left.d \sim 1 \mathrm{~nm}\right)$ the characterization becomes difficult because both the caloric curves and the Lindemann indices fluctuate over wide intervals of temperatures in which the liquid and solid phases coexist in dynamic equilibrium. This well known phenomenon, called dynamic coexistence melting [78] and shown in Figures [6(a) and 6(b), is caused by the multitude of different metastable solid phases present at the nanoscale, which have similar free energies, similar volumes (at constant pressure volumes are allowed to change) and different surface arrangements. The particles are quasi-plastic by continuously changing their state while alternating metastable configurations and liquid states (bi-stability). Thus, the observations of thermal properties inside the dynamic coexistence melting interval $\Delta T_{m}$ are affected by two types of fluctuations: physical, due to coexistence of phases (cannot be avoided), and statistical (can be reduced by increasing the total time of the MD simulation). In particular, the fluctuations of the Lindemann index can be captured by analyzing the standard deviation $\sigma_{L I}$. Figure 6(c) illustrates the phenomenon: by plotting $\sigma_{L I}$ versus $T$, we can estimate the dynamic coexistence melting interval $\Delta T_{m}$, the max-solid and min-liquid points. Pure liquid and solid temperature ranges will be the ones with negligible $\sigma_{L I}$.

Figure 7 shows $T_{m}$ of pure $\mathrm{Fe}$ nanoparticles in the whole range of sizes $\left(N_{F e}=80-1000\right)$ for free and supported clusters. Our results, in agreement with other theoretical [33, 34], computational [13, 35], and experimental studies [26, 27, 28, 29, 30], predict a decrease in the melting temperature inversely proportional to the cluster diameter [85]. The behavior of $T_{m}$ can be described by the model based on the Gibbs-Thomson equation [29, 81, 86] in function of bulk melting temperature $T_{m}^{\text {bulk }}$, effective diameter of the particle $d$, latent heat of melting $\Delta H_{s v}$, and solid-vapor interfacial energy $\gamma_{s v}$ [87].

The melting point of bulk Fe, obtained by extrapolating the fit to $d \rightarrow \infty\left(T_{m}^{b u l k}\left(N_{F e}=\infty\right) \sim 1416 \mathrm{~K}\right)$ is $\sim 20 \%$ below the real one [88], indicating that our Fe-Fe Born Mayer many body potential is slightly underbinding. The systematic shift in melting temperatures should not affect the main conclusions of our study since we are interested in the trends of the liquidus lines in the phase diagrams rather than their precise values. The supported particles considered here have higher melting point than that of the free clusters. In fact, the attractive interaction with the substrate $\left(\mathrm{Al}_{2} \mathrm{O}_{3}\right)$ induces flattening of the particle and increases the effective diameter in agreement with previous studies [15].

Melting temperatures for magic-size clusters $(N=$ $55,147,309,561,923)$ in our range of simulations are shown in the inset of Figure 7 Due to their inherent 


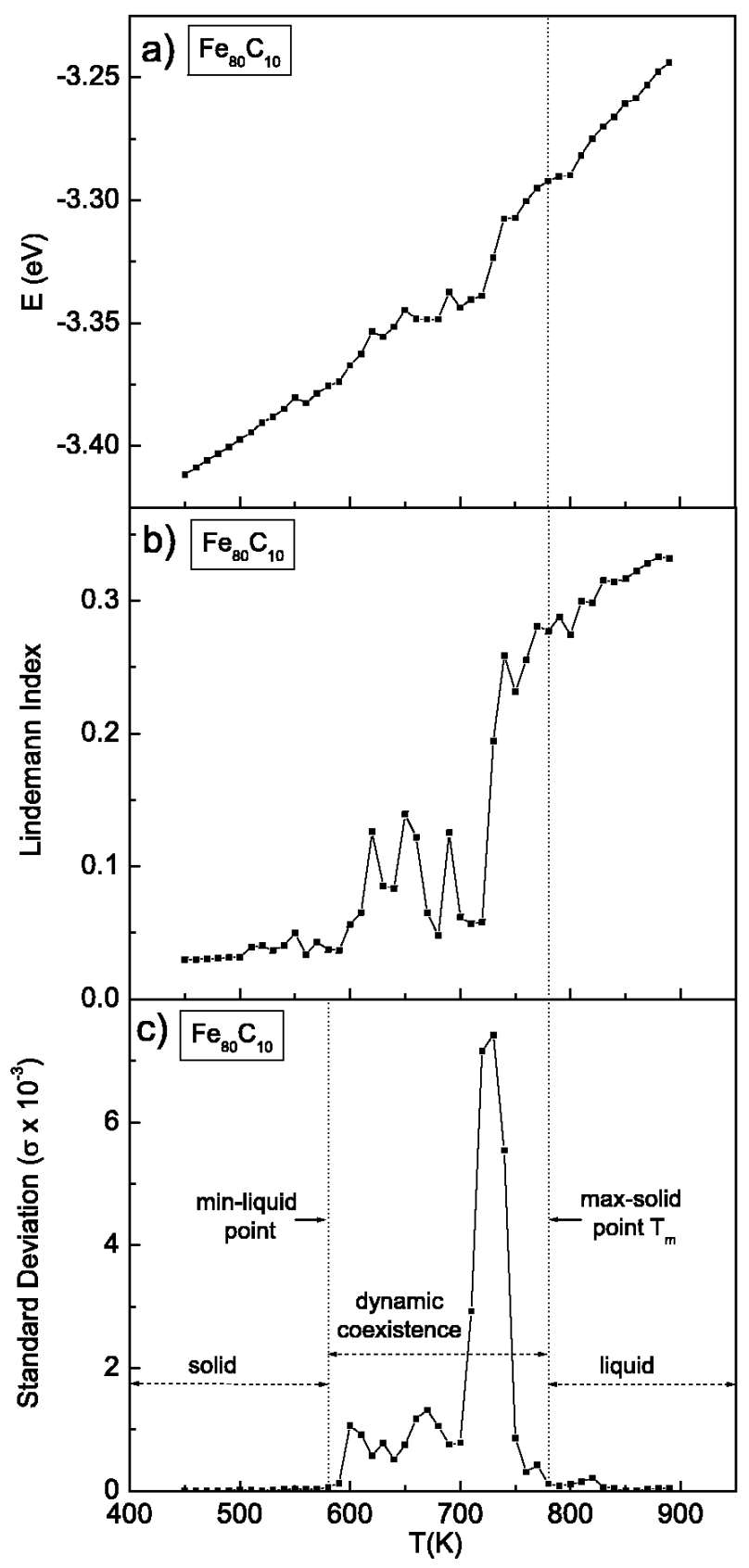

FIG. 6: Melting phenomenon for small nanoparticles $\left(\mathrm{N}_{F e} \sim 80-100\right.$ atoms, $\left.d \sim 1 \mathrm{~nm}\right)$ : a) caloric curve, b) Lindemann index with respect to temperature, c) standard deviation $\sigma_{L I}$ of the Lindemann index to identify the max-solid and min-liquid points.

symmetry (icosahedral or decahedral for small clusters in our case), free magic-size clusters (smaller than $N_{F e}=$ 309) are very stable with melting temperatures higher than that of the non-magic ones. This does not apply for supported clusters. In fact, the presence of substrate interaction changes the magic-size sequence: a cluster of

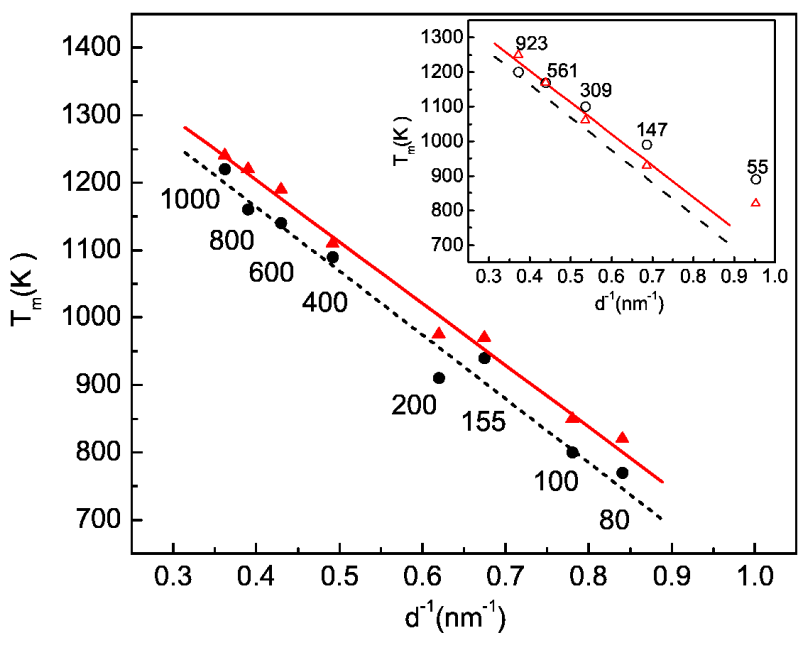

FIG. 7: (color online). Melting temperature versus the inverse of particle diameters. Free non-magic sizes $(\bullet)$ and their linear fit (dashed line); supported non-magic sizes $(\boldsymbol{\Delta})$ and their linear fit (solid line); Supported clusters have higher melting point due to the decreased curvature of the surface [15]. Melting temperatures of free magic sizes $(\circ)$ and supported magic sizes $(\triangle)$ clusters were compared with the linear fit lines of non-magic sizes (inset).

magic-size if free, is not magic if supported (because of the shape and sometimes structural modifications due to interaction with the attractive surface; the phenomenon is addressed in Section (IV)). In summary, the small supported magic-size clusters have lower melting point than the corresponding unsupported ones.

The melting temperature of very small clusters $(N \lesssim$ $80)$ is a function not only of their size but also of their specific structure. It has been shown theoretically that melting temperatures of clusters with several or tens of atoms can be abnormally high, even above the corresponding bulk values [90, 91, 92, 93]. This phenomenon is related to atomistic processes of structure isomerization (geometry reconstruction) [89, 90, 91, 93] or electronic structure change (formation of strong covalent bonds) [92, 94]. Since the thermodynamics of magic-size nanoparticles is significantly influenced by their structure, they experience unusual peculiarities. In this Section we focus our analysis only on the non-magic size ones.

\section{Phase diagram of free and supported Fe-C nanoparticles}

Analysis of phase diagrams. To understand how inclusion of carbon atoms influences the thermal behavior of the catalyst nanoparticles, we determine the melting temperatures as a function of carbon concentration $x^{C}$ ranging from zero to up to $\sim 16 \%$. To appropriately model the nanocatalyst in a nanotube growth process, instead of substituting Fe with $\mathrm{C}$ to increase the concentration of carbon, we add $N_{C}$ atoms to the Fe particle. 
Since $N_{F e}$ remains constant and $N_{C}$ increases, the concentration of carbon is defined as $x^{C} \equiv N_{C} /\left(N_{F e}+N_{C}\right)$. We plot the locus of the max-solid points which represent the liquidus of the $\mathrm{Fe}-\mathrm{C}$ phase diagram. In small $\mathrm{Fe}$ nanoparticles one might expect the average radius to increase noticeably with the addition of a few $\mathrm{C}$ atoms $\left(r^{3} \propto N\right)$ and hence their melting temperature would also increase $\left(\left(T_{m}^{b u l k}-T_{m}\right) / T_{m}^{b u l k} \propto d^{-1}\right)$. However, our tests show that addition of $\mathrm{C}\left(x^{C} \lesssim 16 \%\right)$ does not significantly change the volume of the nanoparticle indicating that $\mathrm{C}$ behaves as an interstitial solute in Fe nanoparticles as it does in bulk Fe [95].

Figure 8 shows phase diagrams $\left(T_{m}\right.$ versus $x^{C}$ in atomic \%) for free and $\mathrm{Al}_{2} \mathrm{O}_{3}$-supported particles with $N_{F e}=80,100$, and 200 based on caloric curve and Lindemann index analysis. All the data sets show a similar trend in function of $\mathrm{C}$ concentration: $T_{m}$ decreases almost linearly at low $x^{C}$ and then increases for all the higher $x^{C}$ considered. The exact functional form is difficult to determine because of the dispersion in the data, however the observed " $V$ "-shape dependence is consistent with that in the bulk $\mathrm{Fe}-\mathrm{C}$ phase diagram [88]. Hence, by using the least square method we approximate the liquidus with a set of two straight lines, the intersection of which gives the eutectic point $\left(x_{\text {eut }}^{C}, T_{\text {eut }}\right)$ [96]. This procedure allows us to estimate this invariant point with an accuracy of $1 \%$ and $12 \mathrm{~K}$ for $x_{\text {eut }}^{C}$ and $T_{\text {eut }}$, respectively.

We observe that as the particle size is reduced, the eutectic point for free and supported nanoparticles moves toward lower temperatures and lower concentrations, indicating that the solubility of $\mathrm{C}$ decreases as well. To the best of our knowledge this phenomenon has never been reported before. Explanation of its origin will require a more detailed study of the behavior of dissolved $\mathrm{C}$ at various concentrations (changes in the distribution of $\mathrm{C}$ across the particle, possible formation of stable carbides etc.).

Implications for carbon nanotube growth. The "V"shape liquidus feature of Fe-C nanoparticles observed in our simulations allows for the VLS interpretation of experimental results for catalytic activity of $\mathrm{Fe}$. For a given temperature above the eutectic temperature $T_{\text {eut }}$, the dissolution of carbon in a metal catalyst initially induces the liquefaction of the particle by lowering its melting temperature [11, 97]. As the catalyst becomes less viscous, the diffusion of carbon in the particle increases. High catalytic activity has been observed with associated liquefied particles during the growth of SWNT by CVD method 97]. Some experiments have shown presence of liquid-like features and liquid layers on nanoparticles [98, 99] before complete melting. These features would enhance the diffusion of carbon and the subsequent melting of the particles. In our case, the nanoparticles are so small that the surface effects on the melting temperature should be dominant. In fact, one cannot distinguish between surface and bulk layers in a particle with 200 atoms of radius $\sim 0.8 \mathrm{~nm}$, which is approximately three close-packed layers.

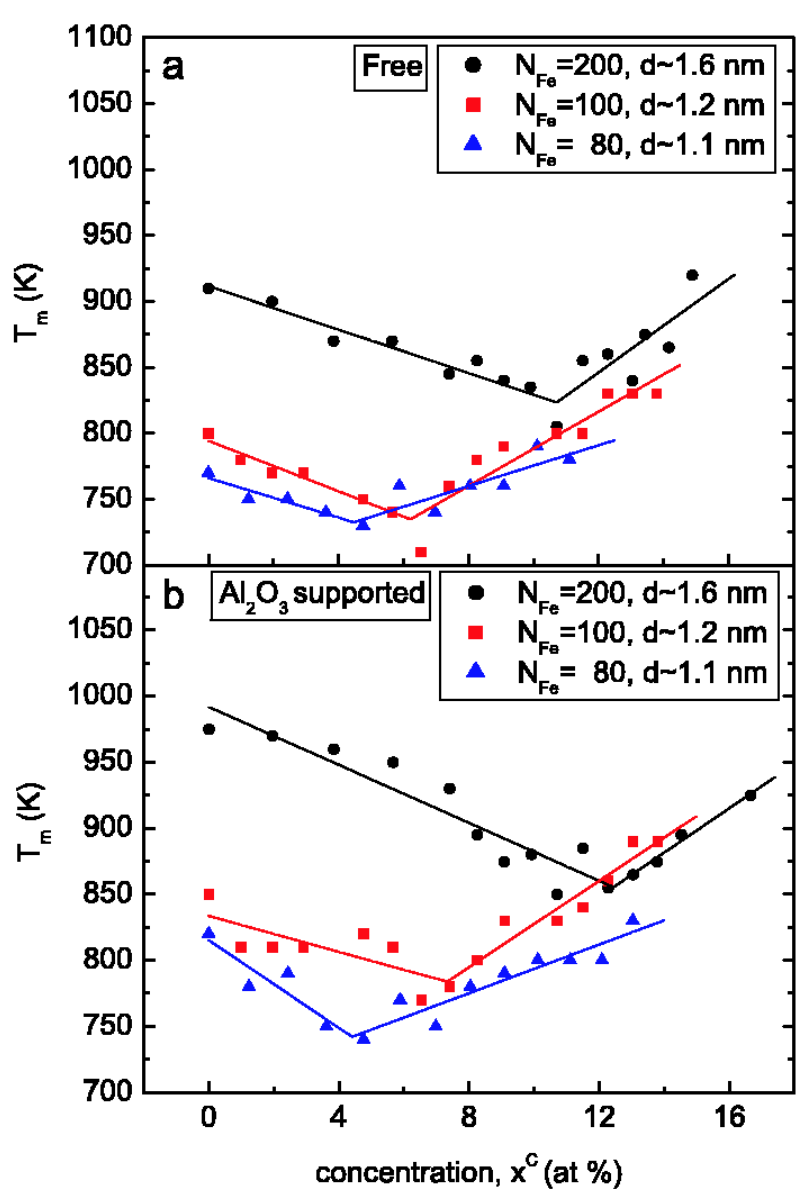

FIG. 8: (color online). Fe-C phase diagrams obtained with addition of C (upto $\sim 16 \%$ ) to particles with $N_{F e}=80,100$, and 200: a) free nanoparticles and b) $\mathrm{Al}_{2} \mathrm{O}_{3}$ supported nanoparticles.

According to the data in Fig. 8, if during CVD experiments the size of the catalyst particle were in a given range $(d \sim 1-2 \mathrm{~nm})$, the smaller Fe nanoparticles $(d \sim 1$ $\mathrm{nm})$ would liquefy earlier then the bigger ones $(d \sim 2$ $\mathrm{nm})$. Consequently, if liquefaction was a prerequisite for catalytic activity, the small particles would begin to produce nanotubes earlier. Experimental verification of this hypothesis is challenging: very small catalyst nanoparticles $(d \cong 1 \mathrm{~nm})$ have a fast rate of coalescence during the reaction and coagulate to form bigger clusters [100, 101]. Following the VLS model, the growth of very small nanotubes using metallic catalyst would be possible only if the reaction temperature were above $T_{\text {eut }}$ (to liquefy the particle) and below the temperature at which particles begin to coalesce. Hence, controlling the diameter of nanotubes grown with CVD in the small size range might be difficult. So far, the smallest reported nanotube $(\mathrm{d}=4$ $\AA$, the most internal tube in a multiwalled nanotube) has been grown by arc-discharge method without any metallic catalyst [102]. 
In the $x^{C}>x_{\text {eut }}^{C}$ region, our results also show that once the dissolved carbon concentration reaches a point where the corresponding melting temperature (from the phase diagram) exceeds the reaction temperature, the particle starts solidifying as a two-phase system composed of solid carbide and Fe-rich liquid. This solidification gradually reduces the average surface mobility of the catalytic species $(\mathrm{Fe})$ and might affect the catalytic activity of the nanoparticle. During the process of nanotube growth, conditions such as sufficient carbon concentration and temperature can induce the formation of stable carbides. Cementite $\left(\mathrm{Fe}_{3} \mathrm{C}\right)$ and other iron carbides have been observed in experiments after the reaction of hydrocarbon or carbon monoxide with iron catalysts $103,104,105]$. It has been reported that such stable carbides act as poison by terminating the growth of nanotube [12, 106].

\section{PECULIARITIES OF SMALL FREE AND SUPPORTED FE CLUSTERS}

In this section we analyze the structural properties of pure small Fe clusters, free and supported on $\mathrm{Al}_{2} \mathrm{O}_{3}$. We find that as the size decreases Fe clusters assume stable polyhedron configurations 107, 108]. The same MD technique described in the previous section is adapted for searching the stable configurations of small clusters $(N \sim 50-80)$. Twenty random configurations are generated per each size and optimized by annealing. The whole annealing process contains $10^{7}$ MD iterations (10 ns). Free and supported clusters are heated to $1200 \mathrm{~K}$ and $1400 \mathrm{~K}$, respectively, before being slowly cooled to $0 \mathrm{~K}$. Among the twenty annealed structures, we consider the lowest energy configuration as the global minimum for each size. For free clusters with $N<72$, the lowest 5 energies are nearly degenerate (inset in Fig. 9) which validates our approach. For bigger particles the found minima might be sub-optimal as the difficulty of finding the global minima rapidly increases.

The minimum energies per atom are fitted to the $E_{\text {ave }}=a+b N^{-1 / 3}$ dependence, where, in the case of spherical particles, the parameters $a$ and $b$ would represent the bulk energy per atom and the surface-creation destabilization energy cost, respectively [109, 110, 111]. Our fit uses only two parameters $(a, b)$ instead of the four of the more general formula $E_{\text {ave }}=a+b N^{-1 / 3}+$ $c N^{-2 / 3}+d N^{-1}$ [109, 110, 111] to avoid overfitting due to the limited number of energy points. We include the best available minima obtained with the aforementioned procedure for bigger $(N=100,120,160$ and 200$)$ clusters to ensure the correct assymptotic behavior for the nanoparticles of large sizes. To capture the size dependence only we exlude sizes $N \sim 55(N=54,55$, and 56) from the fit, because these nanoparticls acheive additional stability by forming highly symmetric icosahedra (see Fig. 9). We obtain $a_{\text {free }}=-4.29 \pm 0.01 \mathrm{eV}, b_{\text {free }}=2.42 \pm 0.03$ $\mathrm{eV}$ and $a_{\text {supp }}=-4.30 \pm 0.01 \mathrm{eV}, b_{\text {supp }}=2.34 \pm 0.02$ $\mathrm{eV}$ for free and supported clusters, respectively. The two values of $a$ are very close to the bulk fcc cohesive energy of $-4.29 \mathrm{eV} /$ atom; the two values of $b$ slightly differ because of the reduced total energy cost to create and modify surface for the supported cluster [15].

Figures 10 and 11 illustrate the differences between the calculated and the fitted energies for free and supported clusters. The prominent negative peaks in Fig. 10 for sizes $N=55,61,64,71,75$, and 77 correspond to the magic size structures in our sequence. The evolution of the nanoparticle configuration with size is depicted in the same picture. By comparing Figures 10 with 11 we observe that the attractive substrate has little or no effect on the internal structure of small clusters $(N \lesssim 70)$. Due to the spherical (or nearly-spherical) arrangements, such small icosahedra are not significantly deformed by attractive substrates, unless the adsorption potentials is comparable to the internal atomic binding energy of the cluster. Big supported particles behaves differently. In fact, as the size increases beyond $N \gtrsim 70$, alumina-supported clusters form fcc arrangements (Fig. 11) with the $\{111\}$ planes at the particle/substrate interface. Elsewhere the particles have simple close-packed facets or local arrangements of them.

Big unsupported particles are different than the supported ones. Unsupported clusters of sizes between $N \sim$ 70 and $N \sim 200$ have decahedra or icosahedra arrangements (multi-twinned structures [112, 113]). Decahedra are more frequent for medium size particles $(N \sim 70$ to $\sim 100$ ) while icosahedra appear more often for bigger sizes $(N \sim 100$ to $\sim 200)$. Figure 10 shows three particular examples of decahedra clusters with $N=71,75$, and 77 . As the size exceeds $N \sim 200$, the particles approach the bulk configuration and the fcc and icosahedra structures tend to become degenerate. For instance, the cluster at $N=500$ is a good example of an fcc particle with ap-

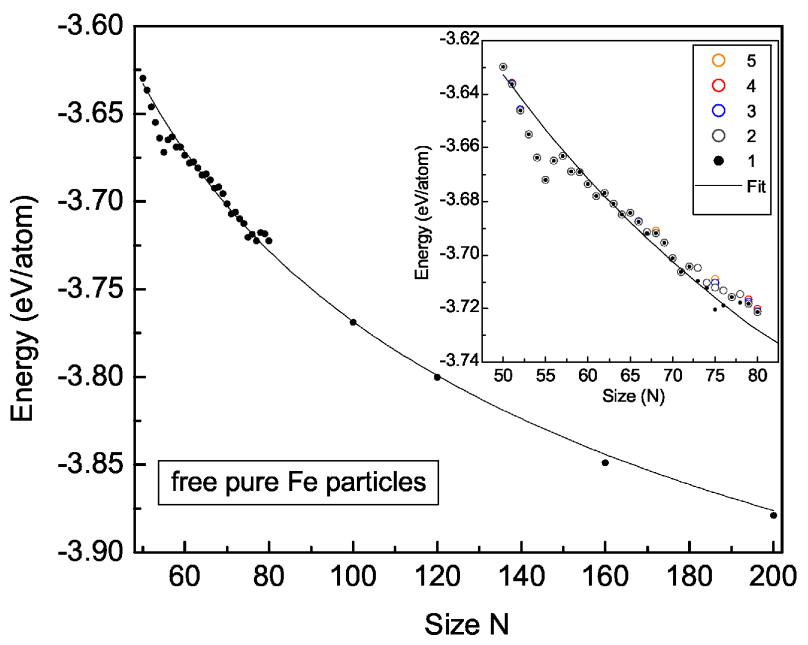

FIG. 9: (color online). The minimum energies of free clusters and the fitting curve using $E_{\text {ave }}=a+b N^{-1 / 3}$. The 5 lowest energies are shown in the insert for cluster's sizes from 50 to 80. 


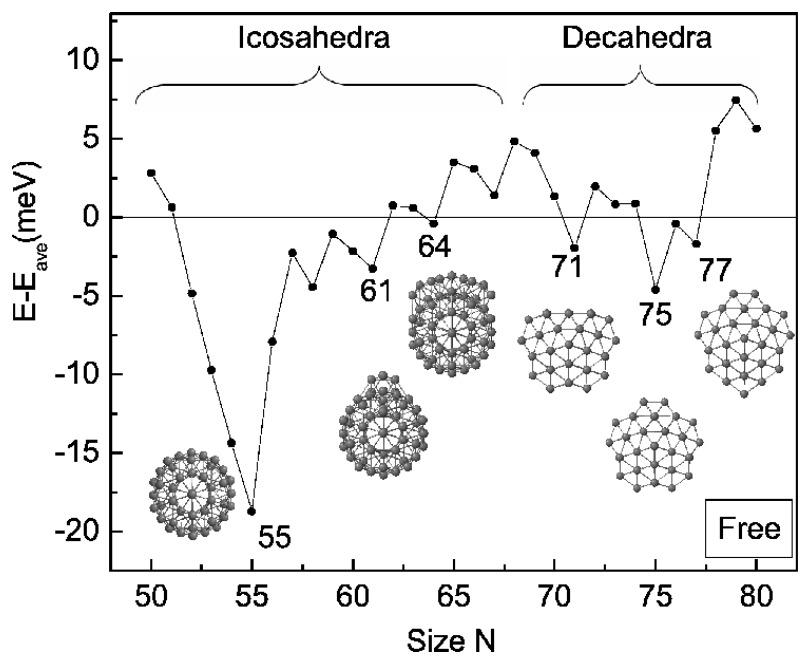

FIG. 10: Energies of best minima for free clusters relative to $E_{\text {ave }}$, with a selection of stable structures. For free clusters, the polyhedron $\rightarrow$ fcc transition is continuous and occurs around $N \sim 200-500$ (not shown).

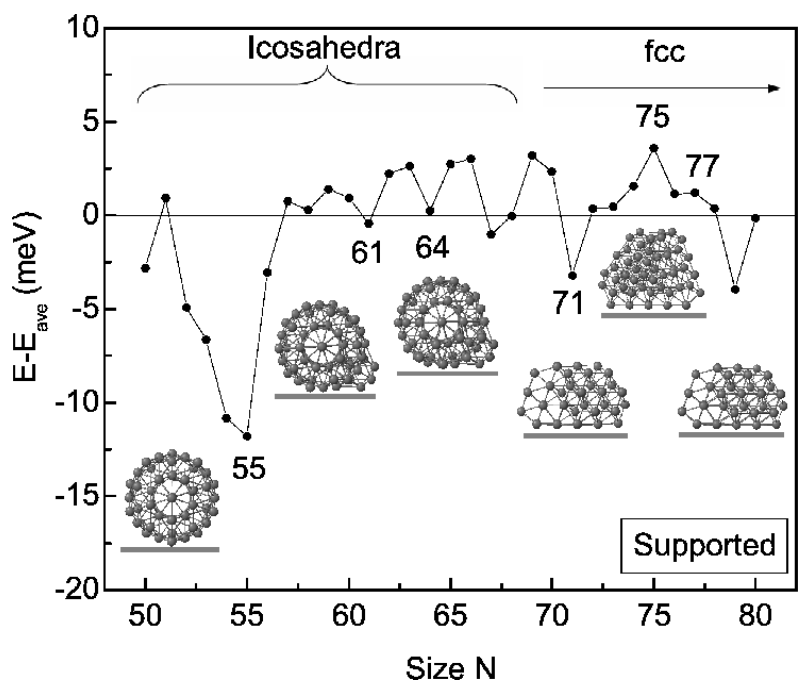

FIG. 11: Energies of best minima for supported clusters relative to $E_{\text {ave }}$, with a selection of structures. For alumina supported clusters, the polyhedron $\rightarrow$ fcc transition occurs around $N \sim 70$.

propriate close-packed faceting and minimal surface-edge reorganization.

The polyhedron $\rightarrow$ fcc transition that occurs at lower sizes for supported clusters can be explained in terms of surface/interface and elastic strain energies [114, 115]. In a groundstate configuration, the former (latter) energy dominates over the other for a small (big) particle. Particles with radii of $r<r_{0}\left(r_{0}\right.$ is the critical size for the polyhedron-fcc transition [116]) tend to form polyhedral structures (icosahedra or decahedra) to minimize the surface/interface energy, while clusters with $r>r_{0}$ will prefer the bulk configuration (fcc) to minimize the elastic strain energy [115]. This is true for free and supported clusters. However, for supported particles, the fcc configuration with flat facets (along the directions of minimum surface energy given by the Wulff plots [117, 118]) has a larger contact area with the substrate than the polyhedra do. Therefore, the attractive substrate interaction reduces the surface/interface energy of fcc the most, resulting in a lower critical $r_{0}$ (even though the overall surface area of the fcc structures could be larger than that of the icosahedra).

Our simulations reveal that the substrate attractive interaction tends to preserve the bulk structure for small particles. This demonstrates that although the Fe- $\mathrm{Al}_{2} \mathrm{O}_{3}$ interaction is relatively weak, it is able to influence the balance between the bulk and surface energies in competing configurations and utlimately determine the particle's ground state. One can also expect to observe this effect in simulations with different models of the Fe-Fe and Fe$\mathrm{Al}_{2} \mathrm{O}_{3}$ interactions, as long as their relative strength is comparable with ours.

\section{CONCLUSIONS}

In this paper we have investigated the behavior of free and alumina-supported Fe-C nanoparticles. We observe interesting phenomena that can be attributed to the presence of the substrate. The main results of the present study can be summarized as follows:

(i) The total Fe binding can be conveniently decomposed into independent $\mathrm{Fe}-\mathrm{Fe}$ and $\mathrm{Fe}-\mathrm{Al}_{2} \mathrm{O}_{3}$ parts: according to our $a b$ initio calculations the two differ by about an order of magnitude. Moreover, the corrugation of the Fe- $\mathrm{Al}_{2} \mathrm{O}_{3}$ interaction is much smaller than the average adsorption energy. This allows us to parameterize the Fe- $\mathrm{Al}_{2} \mathrm{O}_{3}$ interaction as a Morse potential, which includes the deformation energy of the substrate.

(ii) The thermal behavior of pure-Fe particles is simulated with classical MD techniques. We observe the reduction of melting temperature as a function of the diameter, in agreement with the Gibbs-Thomson law. We also show that supported particles have higher melting points than the unsupported ones.

(iii) We calculate the liquidi on the phase diagrams for a range of $\mathrm{Fe}-\mathrm{C}$ nanoparticles $(d \sim 1.1-1.6 \mathrm{~nm})$ and show that they are characterized by the presence of eutectic points in which the eutectic concentration depends on the size of the particles. These phenomena may have important effects on the growth of carbon nanotubes by CVD, as discussed in Section III C.

(iv) We find that the optimized configurations of very small pure Fe clusters (down to $N_{F e} \sim 50$ ) have icosahedron or decahedron structures. Bigger clusters tend to have close-packed configurations. We show that the size at which the cluster undergoes the polyhedron $\rightarrow$ closepacked transition depends on the substrate. In particular, $\mathrm{Al}_{2} \mathrm{O}_{3}$ supported Fe clusters have $N_{t r}^{\mathrm{Al}_{2} \mathrm{O}_{3}} \sim 70$ while free cluster have $N_{t r}^{\text {free }} \sim 200-500$. 
The thermodynamic study of nanoparticles will be extended with future $a b$ initio characterizations carried out in this laboratory, and with experimental investigations performed by our collaborators. This work stimulates more comprehensive studies of the role of substrates on the thermodynamics of small particles, to understand the fundamental factors controlling the catalytic properties of small clusters.

We wish to acknowledge helpful discussions with $\mathrm{T}$. Tokune, E. Mora, H. Duan, A. Rosén, F. Ding, A. Ferrari, L. Boeri, and M. Cole. The authors are grateful for time allocated on the Swedish National Supercomputing facilities. This research was supported by Honda Research Institute USA, Inc.
[1] J. Kong, H. T. Soh, A. M. Cassell, C. F. Quate, and H. Dai, Nature 395, 878 (1998).

[2] C. Ducati, I. Alexandrou, M. Chhowalla, G. A. J. Amaratunga, and J. Robertson, J. Appl. Phys. 92, 3299 (2002).

[3] D. B. Geohegan, A. A. Puretzky, I. N. Ivanov, S. Jesse, and G. Eres, J. Y. Howe, Appl. Phys. Lett. 83, 1851 (2003).

[4] Y. T. Lee and J. Park, Y. S. Choi, H. Ryu, and H. J. Lee, J. Phys. Chem. B 106, 7614 (2002).

[5] A. R. Harutyunyan, B. K. Pradhan, U. J. Kim, G. Chen, and P. C. Eklund, Nano Lett., 2, 525 (2002).

[6] S. Helveg, C. Lspez-Cartes, J. Sehested, P. L. Hansen, B. S. Clausen, J. R. Rostrup-Nielsen, F. Abild-Pedersen, and J. K. Nxrskov, Nature (London) 427, 426 (2004).

[7] F. Abild-Pedersen, J. K. Nxrskov, J. R. RostrupNielsen, J. Sehested, and S. Helveg, Phy. Rev. B 73, 115419 (2006).

[8] S. Hofmann, G. Csanyi, A. C. Ferrari, M. C. Payne, and J. Robertson, Phys. Rev. Lett. 95, 036101 (2005).

[9] S. Hofmann, C. Ducati, J. Robertson, and B. Kleinsorge, Appl. Phys. Lett. 83, 135 (2003).

[10] S. Hofmann, C. Ducati, B. Kleinsorge, and J. Robertson, Appl. Phys. Lett. 83, 4661 (2003).

[11] A. R. Harutyunyan, T. Tokune, and E. Mora. Appl. Phys. Lett. 86, 153113 (2005).

[12] A. R. Harutyunyan, T. Tokune, and E. Mora. Appl. Phys. Lett. 87, 051919 (2005).

[13] F. Ding, K. Bolton, and A. Rosén, J. Vac. Sci. Technol. A 22, 1471 (2004).

[14] F. Ding, A. Rosén, and K. Bolton, Phys. Rev. B 70, 075416 (2004).

[15] F. Ding, A. Rosén, S Curtarolo, and K. Bolton, Appl. Phys. Lett. 88, 133110 (2006).

[16] R. Seidel, G. S. Duesberg, E. Unger, A. P. Graham, M. Liebau, and F. Kreupl. J. Phys. Chem. B 108, 1888 (2004).

[17] A. Fonseca, K. Hernadi, P. Piedigrosso, J. -F. Colomer, K. Mukhopadhyay, R. Doome, S. Lazarescu, L. P. Biro1, Ph. Lambin, P. A. Thiry, D. Bernaerts, and J. B. Nagy, Appl. Phys. A: Mat. Sci. \& Proc. , 67, 11 (1998).

[18] K. Hernadi, Z. Kónya, A. Siska, J. Kiss, A. Oszkó, J. B. Nagy, and I. Kiricsi, Mat. Chem. and Phys. , 77, 536 (2002).

[19] R. S. Wagner and W. C. Ellis, Appl. Phys. Lett. 4, 89 (1964).

[20] M. Audier and M. Coulon, Carbon, 23, 317 (1985).

[21] R. T. K. Baker, M. A. Barber, P. S. Harris, F. S. Feates, and R. J. Waite, J. Catal. 26, 51 (1972).

[22] R. T. K. Baker, P. S. Harris, F. Henderson, and R. B. Thomas, Carbon, 13, 17 (1975).

[23] R. T. K. Baker and P. S. Harris in Chemistry and
Physics of Carbon (Marcel Dekker, New York, 1978), Vol. 14. p. 83.

[24] M. Chen, C. -M. Chen, S. -C. Shi, and C. -F. Chen, Jpn. J. Appl. Phys. bf 42, 614 (2003).

[25] Y. Li, D. Mann, M. Rolandi, W. Kim, A. Ural, S. Hung, A. Javey, J. Cao, D. Wang, E. Yenilmez, Q. Wang, J. F. Gibbons, Y. Nishi, and H. Dai, Nano Lett. 4, 317 (2004).

[26] P. A. Buffat and J. P. Borel, Phys. Rev. A 13, 2287 (1976).

[27] S. L. Lai, J. R. A. Carlsson, and L. H. Allen, Appl. Phys. Lett. 72, 1098 (1998).

[28] K. F. Peters, J. B. Cohen, and Y. W. Chung, Phys. Rev. B 57, 13430 (1998).

[29] S. L. Lai, J. Y. Guo, V. Petrova, G. Ramanath, and L. H. Allen, Phys. Rev. Lett. 77, 99 (1996).

[30] M. Dippel, A. Maier, V. Gimple, H. Wider, W. E. Evenson, R. L. Rasera, and G. Schatz, Phys. Rev. Lett. 87, 095505 (2001).

[31] O. P. Krivoruchko and V. I. Zaikovskii, Kinet. Cataly. 39, 561 (1998).

[32] V. N. Parmon, Catal. Lett. 42, 195 (1996).

[33] R. R. Vanfleet and J. M. Mochel, Surf. Sci. 341, 40 (1995).

[34] H. Reiss, P. Mirabel, and R. L. Wetten, J. Phys. Chem. 92, 7241 (1988).

[35] Y. Qi, T. Cagin, W. L. Johnson, and W. A. Goddard, J. Chem. Phys. 115, 385 (2001).

[36] K. Michaeliana and I. L. Garzon, Eur. Phys. J. D 34, 183 (2005).

[37] A. Aguado and J. M. Lopéz, Phys Rev. B 71, 075415 (2005).

[38] S. K. R. S. Sankaranarayanan, V. R. Bhethanabotla, and B. Joseph, Phys. Rev. B 71, 195415 (2005).

[39] G. Rossi, A. Rapallo, C. Mottet, A. Fortunelli, F. Baletto, and R. Ferrando, Phys. Rev. Lett. 93, 105503 (2004).

[40] J. L. Rodríguez-Lopéz, J. M. Montejano-Carrizales, and M. José-Yacamán, Appl. Surf. Sci. 219, 56 (2003).

[41] The highly disperse unstable solutions are oversaturated with respect to the thermodynamic equilibrium "graphite $\leftrightarrow$ solution of carbon in the metal" which is kinetically slow to reach starting from the mixture "amorphous carbon $\leftrightarrow$ oversaturated solution of carbon". The stability of the disperse solutions should be compared to the latter mixture and not to the thermodynamic equilibrium (see Refs. 31] and [32]).

[42] A. C. Ferrari and S. Curtarolo, Private comunications regarding reference number \#19 of Ref. [8], which instead should point to Ref. 13]. In the latter work, the eutectic temperature is studied only for one particle size (Fig. 3 of Ref. 13]) and not in function of size and sub- 
strate as in our manuscript.

[43] S. -P. Huang, D. S. Mainardi, and P. B. Balbuena, Surf. Sci. 545, 163 (2003).

[44] A. Antonelli, S. N. Khanna, and P. Jena, Phys. Rev. B 48, 8263 (1993).

[45] F. H. Streitz and J. W. Mintmire, Phys. Rev. B 50, 11996 (1994);

[46] X. W. Zhou, H. N. G. Wadley, J. -S. Filhol, and M. N. Neurock, Phy. Rev. B 69, 035402 (2004).

[47] C. Verdozzi, D. R. Jennison, P. A. Schultz, and M. P. Sears, Phys. Rev. Lett. 82, 799 (1999).

[48] J. Ahn and J. W. Rabalais, Surf. Sci. 388, 121 (1997).

[49] J. Toofan and P. R. Wilson, Surf. Sci. 401, 162 (1998).

[50] P. Guenard, G. Renaud, A. Barbier, and M. GautierSoyer, Surf. Rev. Lett. 5, 321 (1988).

[51] G. Kresse and J. Hafner, Phys. Rev. B 47, 558 (1993); Phys. Rev. B 49, 14251 (1994).

[52] G. Kresse and J. Furthmuller, Phys. Rev. B 54, 11169 (1996); Comput. Mater. Sci. 6, 15 (1996).

[53] P. E. Blochl, Phys. Rev. B 50, 17953 (1994).

[54] G. Kresse and D. Joubert, Phys. Rev. B 59, 1758 (1999).

[55] J. P. Perdew, K. Burke, and M. Ernzerhof, Phy. Rev. Lett. 77, 3865 (1996).

[56] J. D. Pack and H. J. Monkhorst, Phys. Rev. B 13, 5188 (1976); 16, 1748 (1977).

[57] H. d'Amour, D. Schiferl, W. Denner, H. Schulz, and W. B. Holzapfel, J. App. Phys. 49, 4411 (1978).

[58] P. Villars, K. Cenzual, J. L. C. Daams, F. Hulliger, T. B. Massalski, H. Okamoto, K. Osaki, A. Prince, and S. Iwata, Crystal Impact, Pauling File. Inorganic Materials Database and Design System, Binaries Edition, ASM International, Metal Park, OH (2003).

[59] M. Guillope and B. Legrand, Surf. Sci. 215, 577 (1989).

[60] J. Stanek, G. Marest, H. Jaffrezic, and H. Binczycka, Phys. Rev. B 52, 8414 (1995).

[61] K. Matsunaga, T. Sasaki, N. Shibata, T. Mizoguchi, T. Yamanoto, and Y. Ikuhara, Phys. Rev. B 74, 125423 (2006).

[62] P. M. Morse, Phys. Rev. 34, 57 (1929).

[63] L. J. Lewis, P. Jansen, and J.-L. Barrat, Phys. Rev. B 56, 2248 (1997).

[64] M. Wautelet, J. P. Dauchot, and M. Hecq, Mat. Sci. Eng. C 23, 187 (2003).

[65] C. Mottet, G. Rossi, F. Baletto, and R. Ferrando, Phys. Rev. Lett. 95, 035501 (2005).

[66] L. Verlet, Phys. Rev. 159, 98 (1967).

[67] L. Verlet, Phys. Rev. 165, 201 (1967).

[68] H. J. C. Berendsen, J. P. M. Postma, W. van Gunsteren, A. DiNola, and J. R. Haak, J. Chem. Phys. 81, 3684 (1984).

[69] S. Nose, J. Chem. Phys. 81, 511 (1984)

[70] S. Nose, Molec. Phys. 52, 255(1984).

[71] W. G. Hoover, Phys. Rev. A 31, 1695 (1985).

[72] S. A. Adelman and J. D. Doll, J. Chem. Phys. 64, 2375 (1976).

[73] B. L. Holian, A. F. Voter, and R. Ravelo, Phys. Rev. E 52, 2338 (1995).

[74] Y. Hu and S. B. Sinnott, J. Comp. Phys. 200, 251 (2004).

[75] R. A. Johnson, Phys. Rev. 134, A1329 (1964).

[76] F. Ding, K. Bolton, and A. Rosen, J. Phys. Chem. B 108, 17369 (2004).

[77] L. A. Girifalco, J. Chem. Phys. 96, 858 (1992).
[78] S. Alavi and D. L. Thompson, J. Phys. Chem. A 110, 1518 (2006).

[79] X. Zhu, X. You, R. Xiong, and Z. Zhou, Chem. Phys. 269, 243 (2001).

[80] F. A. Lindemann, Phys. Z. 11, 609 (1910).

[81] C. H. P. Lupis, Chemical Thermodynamics of Materials, North-Holland, New York (1983).

[82] D. A. McQuarrie, Statistical Mechanics, Harper \& Row, New York (1976).

[83] P. Labastie and R. L. Whetten, Phys. Rev. Lett. 65, 1567 (1990).

[84] S. Iijima and T. Ichihashi, Phys. Rev. Lett. 56, 616 (1986).

[85] J. J. Thomson, App. of Dynamics to Phys. and Chem. (London, Macmillan), 1888.

[86] K. J. Hanszen, Z. Phys. 157, 523 (1960).

[87] F. Celestini, R. J.-M. Pellenq, P. Bordarier, and B. Rousseau, Z. Phys. D 37, 49 (1996).

[88] T. B. Massalski (Ed.), Binary Alloy Phase Diagrams, ASM International, Metals Park, OH (1992).

[89] B. Soule de Bas, M. J. Ford, and M. B. Cortie, J. Phys.: Cond. Matter 18, 55 (2006).

[90] A. A. Shvartsburg and M. F. Jarrold, Phys. Rev. Lett. 85, 2530 (2000).

[91] F. -C. Chuang, C. Z. Wang, S. Ogut, J. R. Chelikowsky, and K. M. Ho, Phys. Rev. B 69, 165408 (2004).

[92] S. Chacko, K Joshi, D. G. Kanhere, and S. A. Blundell Phys. Rev. Lett. 92, 135506 (2004).

[93] G. A. Breaux, R. C. Benirschke, T. Sugai, B. S Kinnear, and M. F. Jarrold. Phys. Rev. Lett. 91, 215508 (2003).

[94] K. Joshi, D. G. Kanhere, and S. A. Blundell. Phys. Rev. B 66, 155329 (2002).

[95] B. J. Lee, Act. Mater. 54, 701 (2006).

[96] We use the least square method to determine the four parameters of the fitting function $\left(x_{\text {eut }}^{C}, T_{\text {eut }}\right.$, and the slopes of the two straight lines).

[97] A. R. Harutyunyan, E. Mora, T. Tokune, K. Bolton, A. Rosen, A. J. Epstein, A. Jiang, N. Awasthi, and S. Curtarolo, (submitted)

[98] F. Ercolessi, W. Andreoni, and E. Tosatti, Phys. Rev. Lett. 66, 911 (1991).

[99] R. Kofman, P. Cheyssac, A. Aouaj, Y. Lereah, G. Deutscher, T. Ben-David, J. M. Penisson, and A. Bourret, Surf. Sci. 303, 231 (1994).

[100] A. R. Harutyunyan, T. Tokune, E. Mora, J.-W. Yoo, and A. Epstein, J. App. Phys. 100, 044321 (2006).

[101] A. Wadhawan, D. Garrett, and J. M. Perez, App. Phys. Lett. 83, 2683 (2003).

[102] L.-C. Qin, X. Zhao, K. Hirahara, Y. Miyamoto, Y. Ando, and S. Iijima, Nature 408, 50 (2000).

[103] A. Sacco, P. Thacker, T. N. Chang, and A. T. S. Chiang, J. Catal. 85, 224 (1984).

[104] A. Oberlin, M. Endo, and T. Koyama, J. Cryst. Growth 32, 335 (1976).

[105] A. J. H. M. Kock, P. K. de Bokx, E. Boellard, W. Klop, and J. W. Geuss, J. Catal. 96, 468 (1985).

[106] J. Gavillet, A. Loiseau, C. Journet, F. Willaime, F. Ducastelle, and J. C. Charlier, Phys. Rev. Lett. 87, 275504 (2001).

[107] D. J. Wales, Science, 271925 (1996).

[108] J. P. K. Doye and D. J. Wales, Phys. Rev. Lett. 865719 (2001).

[109] J. W. Lee and G. D. Stein, J. Phys. Chem. 91, 2450 
(1987).

[110] J. A. Northby, J. Xie, D. L. Freeman, and J. D. Doll, Z. Phys. D 1269 (1989).

[111] J. Xie, J. A. Northby, D. L. Freeman, and J. D. Doll, J. Chem. Phys. 91612 (1989).

[112] S. Ino, J. Phys. Soc. Jap. 21, 346 (1966).

[113] S. Ino and S. Ogawa, J. Phys. Soc. Jap. 22, 1365 (1967).

[114] S. Ino, J. Phys. Soc. Jap. 27, 941 (1969).

[115] R. L. Johnston, Phil. Trans. R. Soc. Lond. A, 356, 231 (1998).

[116] $E_{\text {total }}=E_{\text {cohesive }}+A r^{2}+B r^{3}$, where $A$ and $B$ are the surface and elastic strain energy coefficients, respec- tively, and $r$ is the average radius of the particle. Assuming a constant cohesive energy for clusters of the same size, the polyhedron $\rightarrow$ fcc transition occurs at a critical radius $r_{0}=\left(A_{f c c}-A_{\text {poly }}\right) /\left(B_{\text {poly }}-B_{f c c}\right)$ because of the inherent structural relationships $A_{\text {poly }}<A_{f c c}$ and $B_{\text {poly }}>B_{\text {fcc }}$ [114, 115].

[117] G. Wulff, Z. Krist. 34, 449 (1901).

[118] C. Herring, Structure and Properties of Solid Surfaces (R. Gomer and C. S. Smith, ed.). University of Chicago Press, Chicago, 1952. 